\title{
Patients' perspectives of the quality of tuberculosis treatment services in South Ethiopia
}

\author{
Belay Mergya Eticha ${ }^{1}$, Alemayehu Atomsa ${ }^{2}$, BirtukanTsehaineh ${ }^{2}$, Tezera Moshago Berheto ${ }^{3, *}$ \\ ${ }^{1}$ Disease Prevention and Control Coordinator, Hadiya Zone Health Department, Hosanna, Ethiopia \\ ${ }^{2}$ Department of Epidemiology, Jimma University, Jimma, Ethiopia \\ ${ }^{3}$ Department of Public Health, Mizan College of Health Sciences, Mizan, Ethiopia
}

\section{Email address:}

moshagot1@gmail.com (T. M. Berheto), belaymergya@gmail.com (B. M. Eticha),nubial2008@yahoo.com (A. Atomsa), birtukan_t@yahoo.com (B. Tsehaineh)

\section{To cite this article:}

Belay Mergya Eticha, Alemayehu Atomsa, BirtukanTsehaineh, Tezera Moshago Berheto: Patients' Perspectives of the Quality of Tuberculosis Treatment Services in South Ethiopia. American Journal of Nursing Science. Vol. 3, No. 4, 2014, pp. 48-55.

doi: 10.11648/j.ajns.20140304.12

\begin{abstract}
Background: Observing quality from the patient's perspective is of paramount importance for making the service more responsive to patients. However, little is known about the quality of tuberculosis treatment service from the patient's perspective in Ethiopia. This study was carried out to assess the quality of tuberculosis treatment services from the patient's perspective in South Ethiopia's public health facilities. Methods: A facility-based, cross-sectional study design was employed, and data were collected from February to March, 2012. A QUOTE-TB tool validated for East African countries was used to collect data from a sample of 370 tuberculosis patients receiving treatments at public health facilities. The study participants were allocated proportionally to the number of client receives the service, and the relative importance of the aspects and perception of the quality of their received care was scored. Combining the relative importance and actual performance scores derived a measure of service quality. Analyses were performed using SPSS and statistical significance was set at a p-value less than 0.05. Results: The accommodation aspects of the facilities, patient-provider interactions, health information and communication, and availability of care had low quality impact scores. The highest service quality scores were for TB-HIV integration and cost of treatment. Shorter waiting times $(\beta=-1.85)$, routine observation, and checking the daily TB drug intake $(\beta=-1.26)$ and treatment by the same health provider $(\beta=1.13)$ independently predicted overall patient satisfaction. Conclusion: There were notable differences between TB patients 'expectations and what they actually received in terms of accommodation, availability, patient-provider interactions, health information, and communication, which were identified as being of inadequate quality. Significant effort is needed to improve the quality of TB care with respect to these particular aspects from the perspectives of patients. Individuals caring for patients with TB in the health service should address these areas of care in order to enhance TB treatment services and satisfy patients.
\end{abstract}

Keywords: Tuberculosis, Quality of Treatment Service, Satisfaction

\section{Background}

Tuberculosis (TB) remains a major cause of morbidity and mortality in many developing countries [1, 2]. Approximately $95 \%$ of all TB cases and $99 \%$ of deaths occur in developing countries, with the greatest burden in sub-Saharan Africa and South East Asia [3]. In addition, TB hinders socioeconomic development, because $75 \%$ of people with TB are within the economically productive age group of 15-54 years [1, 3].

The quality of healthcare is a public health concern in many countries, including in the developing world [2, 4]. It is also an issue addressed as a component of the Ethiopia Health Sector Development Program (HSDP)and an intrinsic part of the program [5]. Successful tuberculosis treatment depends on more than chemotherapy and requires specific clinical and social frameworks based on an individual patient's circumstances [6].

In Ethiopia, patients have free access to diagnosis and TB treatment services from public health facilities. TB treatment under directly observed treatments (DOTs) has two phases: an intensive (initial) phase, which comprises the first 8 weeks for new cases and twelve weeks for re- 
treatment cases, and a continuation phase of 4 - 6 months immediately following the intensive phase [7].

Poor adherence to treatments by patients arises from the interaction of multiple factors affecting the quality of $\mathrm{TB}$ care $[8,9]$. Previous studies have identified several health service delivery factors that impact on adherence, including ineffective communication, poorly supervised health staff incapable of dealing with minor illnesses, and reduced access to TB care units $[8,10,11]$.

Patient views and judgments on health care services are vital parts of quality assurance in health care [12]. Managers or health professionals often formulate judgments based on the quality of care. However, patient views on the quality of health care differ from the views of health care professionals, managers, and policy makers [11, $12]$.

Observing quality from a patient's perspective is best way to empower tuberculosis patients and their communities' and contribute to achieving the Millennium Development Goals (MDGs). However, while assessing quality from the patient's perspective is of paramount importance, it has not been addressed well to date.

\section{Materials and Methods}

\subsection{Study setting and Design}

A facility-based cross sectional descriptive study was conducted in Hadiya zone public health facilities. The Hadiya zone, $230 \mathrm{~km}$ from Addis Ababa, is in the South Nation Nationality People Regional State of Ethiopia [13]. During the time of the study, one hospital and 55 health centers were providing TB services. Of these, only 37 of the facilities had registered TB patients during data collection period.

\subsection{Participants}

The study population included adult patients (aged $\geq 18$ years) who used the TB treatment service for at least for three weeks prior to the data collection period at public health facilities. The sample size was determined using a single population proportion formula

$$
\mathrm{n}=\frac{\left(\mathrm{Z}_{\left(\frac{\alpha}{2}\right)}\right)^{2} \mathrm{P}(1-\mathrm{P})}{\mathrm{d}^{2}}
$$

which considered the following parameters: the proportion of clients satisfied to provider patient interaction was $62.6 \%$ [14], with 5\% marginal error and a 95\% confidence intervals. Considering a $10 \%$ possible non-response rate, this value became 396 .

Thirty-seven health facilities providing DOTs services were included for selection of study participants. The heath facilities providing TB treatment services were stratified into hospitals and health centers. Then, the sample was distributed to the health facilities proportional to the size of their patient populations. Finally, using the TB unit register as a frame, patients who fulfilled the inclusion criterion were included in the study by simple random sampling.

\subsection{Measurements}

Data were collected by trained high school graduate personnel using a pre-tested structured questionnaire, which included questions about socio-demographic characteristics, 32 items in seven criteria of TB care quality from the patient's perspective adopted from the QUOTETB tool validated for East African countries, and overall satisfaction with care (4 items) $[15,16]$. The quality of care aspects were formulated as importance and performance statements in the questionnaire. Respondents were asked to rate the aspects with respect to health care providers. Importance was measured using a 4-point Likert scale and the performance aspect required a dichotomized (yes or no) response.

The importance aspect scores, which have values between 0 and 10, were calculated using the following categories: 1="not important" (score 0), 2="fairly important" (score 3), 3="important" (score 6), and 4="extremely important" (score 10) [15-17]. The performance aspect categories were converted into either "yes" (score 0) or "no" (score 1) responses, and were expressed as a proportion of patients who reported that the aspect was absent. In the best situation, all quality of care aspects would receive a 0 performance score, indicating that no aspect was reported as absent. Scores between 0 and 0.10 meant little improvements was needed and was, therefore, regarded as optimal. Aspects of care with room for improvement from a patient's perspective or low quality aspects were identified as a quality impact score/value below 9 and performance score above 0.10 . The concept "quality of health care from patients" perspectives" operationalize as the product of importance and performance using the Netherlands Institute for Health Services Research methodology $[15,16]$. Accordingly, the formula: $\mathrm{Qij}=\mathrm{Iij} \times \mathrm{Pij}$ was used where the quality improvement score (Q) on a health service (j) by an individual patient (i) is equal to the importance score (I) multiplied by the performance score $(\mathrm{P})$.

\subsection{Service Quality (Quality Impact)}

The service quality (quality impact) was defined as $10-$ (importance $\times$ performance). Quality-impact factors can be interpreted as a weighted performance score. Theoretically, a quality impact score can vary from 0 to 10 , where a score of 10 indicated that $0 \%$ of patients reported the absence of this particular aspect, showing the best possible quality of care. However, a 0 score indicated that all respondents thought that this aspect was important, and $100 \%$ of the patients reported that it needed improvement or was of the worst quality [15-17].

\subsection{Statistical Analysis}

Data were double entered using Epi-data version 3.1. For 
analysis, the data were exported to SPSS version 16.0. Descriptive statistics and mean scores were used to summarize data. Multivariate linear regression analysis was used to identify independent predictors of satisfaction. pvalues less than 0.05 were considered statistically significant.

\subsection{Ethical Considerations}

The ethical issues of this study were reviewed and approved by the ethical review committees of Jimma University. Oral consent was obtained from each participant before the study interviews.

\section{Results}

\subsection{Socio-Demographic Characteristics}

From the entire 396 TB patients sampled, 370 (93.4\%) completed the interview. The majority, 342 (92.4\%) of participants, was from public health centers and $28(7.6 \%)$ individuals were from the hospital. A total of $52 \%$ of respondents were male and $112(32.4 \%)$ respondents were between 25 and 34 years of age, followed by the 35 to 49 age category, which accounted for $90(24.5 \%)$ individuals. The majority of respondents, 277 (74.9\%), resided rurally, and $213(62.4 \%)$ were married (Table1).

Table 1. Socio-demographic characteristics of TB patients in South Ethiopia, March 2012.

\begin{tabular}{|c|c|c|c|c|}
\hline \multirow{2}{*}{ Background characteristics } & & \multicolumn{2}{|l|}{ Type of facility } & \multirow{2}{*}{ Total N (\%) } \\
\hline & & Health Center N (\%) & Hospital N (\%) & \\
\hline \multirow{2}{*}{ Sex } & Male & $183(53.5)$ & $10(35.7)$ & $193(52.0)$ \\
\hline & Female & $159(46.5)$ & $18(64.3)$ & $177(48.0)$ \\
\hline \multirow{6}{*}{ Age (completed years) $(\mathrm{N}=368)$} & $18-19$ & $50(14.7)$ & $2(7.1)$ & $52(14.1)$ \\
\hline & $20-24$ & $47(13.8)$ & $7(25)$ & $54(14.7)$ \\
\hline & $25-34$ & $128(37.7)$ & $8(28.6)$ & $136(37)$ \\
\hline & $35-49$ & $82(24.1)$ & $8(28.6)$ & $90(24.5)$ \\
\hline & $50-54$ & $13(3.8)$ & $3(10.7)$ & $16(4.3)$ \\
\hline & $55^{+}$ & $20(5.9)$ & $0(0)$ & $20(5.4)$ \\
\hline \multirow{5}{*}{ Education level } & Illiterate & $104(30.4)$ & $2(7.1)$ & $106(28.6)$ \\
\hline & Able to read and write & $33(9.6)$ & $1(3.57)$ & $34(9.3)$ \\
\hline & Grade $1-4$ & $49(14.3)$ & $2(7.1)$ & $51(13.8)$ \\
\hline & Grade $5-8$ & $77(22.5)$ & $10(3.57)$ & $87(23.5)$ \\
\hline & Grade $9^{+}$ & $79(23.2)$ & $13(46.4)$ & $92(24.8)$ \\
\hline \multirow{2}{*}{ Residence } & Urban & 68 (19.9) & $25(89.3)$ & $93(25.1)$ \\
\hline & Rural & $274(80.1)$ & $3(10.7)$ & $277(74.9)$ \\
\hline
\end{tabular}

\subsection{Patient-Reported Measures of TB Treatment Service Quality}

\subsubsection{Importance Rates}

Not all quality aspects of TB treatment services included in this study were valued equally in importance by TB treatment attendees. Some quality aspects were highly valued, whereas others were judged less important or even unimportant by some patients. The mean importance scores for aspects of quality of care in public health centers and hospitals were $8.8 \pm 1.4$ and $8.3 \pm 2.3$, respectively.

The top five importance scores in aspects of TB care at the public health centers were:1) "Drugs should be available when required"(importance score 9.7); 2) "Should not have difficulties in obtaining TB services because of language"(importance score 9.6); 3) "TB services should be available during working hours"(importance score 9.6); 4) "Safe drinking water should be available"(importance score 9.6); and 5) "Health providers should inform about the link between TB and HIV"(importance score 9.5).

While in hospital, the top importance scores in quality of TB care were "Privacy should be respected during examination", "Health provider should show care and compassion", "Health providers should have sufficient time to discuss your problems", and "TB services should be free of charge", all with an equal maximum importance score of 10 .
In contrast, the aspects of TB care with the lowest importance scores were: "Without referral to another health unit for TB services" (importance score of 2.13) and an importance score of 0 for public health centers and hospitals for this aspect of TB care. In addition, hospitals that "Pay a tip in order to obtain TB services" had an importance score of 2.5.

\subsubsection{Performance Component}

The health care providers 'performance was rated by the patients who had attended a specific healthcare service. The performance scores represent the proportion of patients who reported that particular aspects were not performed as stated. The public health centers, which had the highest performance on aspects of TB care, were scored as follows: "Safe drinking water available" (performance score of 0.40);"Waiting time before being served" (performance score of 0.35); and "Attended to by the same health provider" (performance score of 0.39). Concerning hospitals, "Attended to by the same health provider" had a performance score of 0.41 , which was the highest performance score.

Conversely, the aspects of TB care with the lowest performance scores were: "The service hours of facility convenience" (performance score of 0.01 for public health centers and 0 for the hospital). In addition, "Drugs available when required "had performance scores of 0.02 and 0.00 for public health centers and the hospital, 
respectively. Moreover, "Paid for regular TB service" had performance scores of 0.01 and 0.00 for public health centers and the hospital, respectively.

\subsubsection{Inadequate Quality Aspect of TB Care from the Patient's Perspective}

Hospitals where the same health care provider attended to patients had a low quality impact score among available dimensions of care, scoring 3.54. This value was based on an importance score of 6.54 with a corresponding performance score of 0.41 and indicates that $41 \%$ of the attendees in hospital were not attended to by the same health care provider. This score also demonstrates that their needs were not met by the performance of this health facility.

Concerning the information and education dimension, health providers who informed patients about the importance of observed treatments had a low quality impact score of 6.40. This value is based on an importance score of 6.46 with a corresponding performance score of 0.40 ; this performance score indicates that health care providers did not inform $40 \%$ of the attendees in hospital about the importance of the treatment.

Similarly, in relation to patient-provider interaction, health providers who discussed how to deal patients' problems and who respected privacy during examinations had low quality impact scores of 8.93 and 7.86 , respectively. These scores showed that the patients' needs were not met by the performance of this health facility (Table 2). In public health centers; acceptable waiting times had low quality impact scores of 8.28 among the availability dimension of care in health centers. This value is based on an importance score of 6.99 with a corresponding performance score of 0.35 , indicating that $35 \%$ of health center attendees had not been served within an acceptable waiting time. Similarly, in this dimension of care, patients being attended to by the same health care provider had a quality impact score of 6.35. A treatment observer checking on daily intake of TB drugs had a quality impact score of 8.74, which was a low quality impact score among the availability dimension of TB care in health centers. These scores demonstrate that the patients 'needs were not met by the performance of these health facilities or providers, since all performance scores were above 0.1 and the impact values were less than 9 .

Concerning infrastructure or accommodation of facilities, the availability of safe drinking water had a quality impact score of 6.34 , which is based on a weighted importance score of 9.57 and a corresponding performance score of 0.40 , and indicates that $40 \%$ of attendees did not get safe drinking water at their treatment centers. These scores demonstrate that the patients' needs were not met by the performance of the health facilities. Furthermore, all performance scores were above 0.1 withan impact value less than 9. Similarly, facility cleanliness was scored as being of low quality (Table 3).

Table 2. Inadequate quality of TB treatment service from the patient's perspective in Hospital, South Ethiopia, March 2012.

\begin{tabular}{|c|c|c|c|c|}
\hline \multirow{2}{*}{ Aspect category } & \multirow{2}{*}{ Quality of TB care aspect } & \multicolumn{3}{|l|}{ Quality score } \\
\hline & & Performance & Importance & Impact \\
\hline Availability accessibility & Attended by the same care provider & .41 & 6.54 & 3.54 \\
\hline Information and education & Care providers inform about the importance of observed treatment & .40 & 6.46 & 6.40 \\
\hline \multirow{2}{*}{ Patient provider Interaction } & Care providers discuss how to deal with problems & .11 & 9.43 & 8.93 \\
\hline & Privacy respected during examination & .21 & 10.0 & 7.86 \\
\hline
\end{tabular}

Table 3. Inadequate quality of TB treatment service from the patient's perspective in public health centers, South Ethiopia, March 2012.

\begin{tabular}{|c|c|c|c|c|}
\hline \multirow{2}{*}{ Aspect category } & \multirow{2}{*}{ Quality of TB care aspect } & \multicolumn{3}{|l|}{ Quality score } \\
\hline & & Performance & Importance & Impact \\
\hline \multirow{6}{*}{ Availability } & Acceptable waiting times & .35 & 6.99 & 8.28 \\
\hline & Attended to by the same health provider & .39 & 9.00 & 6.35 \\
\hline & Difficulty because of language barrier & .25 & 9.61 & 7.66 \\
\hline & Facility offer services to examine sputum & .14 & 9.15 & 8.64 \\
\hline & Treatment observer checking daily intake of TB drugs & .14 & 9.26 & 8.74 \\
\hline & Costs for transport prevent you from getting to TB facility & .19 & 6.68 & 8.34 \\
\hline \multirow{4}{*}{ Patient provider Interaction } & Care providers discuss how to deal with problems & .14 & 8.99 & 8.96 \\
\hline & Care provider listens carefully & .20 & 9.26 & 8.21 \\
\hline & TB-related discrimination & .21 & 8.51 & 8.24 \\
\hline & Privacy respected during examination & .20 & 8.84 & 8.33 \\
\hline \multirow{3}{*}{ Infrastructure } & Cleanliness of the facility & .12 & 9.45 & 8.91 \\
\hline & Availability of safe drinking water & .40 & 9.57 & 6.34 \\
\hline & Enough comfortable benches /weighting area / & .20 & 9.36 & 8.12 \\
\hline
\end{tabular}

\subsubsection{Optimal Quality Aspect of TB Care from the Patient's Perspective}

Optimal quality of care indicated that patient needs were almost completely met by the performance of the health care providers or health facilities. In these cases, reports or experiences on a particular aspect of inadequate care performance were between 0 and 0.10 , with an impact quality score less than 1 .

In hospitals, for the availability dimension of TB service, no treatment attendee claimed any inadequate performance. With respect to the health information and education 
dimension of TB care, informing patients about how TB can be cured and the possible side effects of TB drugs had a quality impact value above nine. These values are based on importance scores of 6.46 and 6.86 , respectively. Also, the corresponding performances scored 0.04 , which indicated that $4 \%$ of the respondents did not receive these particular services. These scores demonstrate that patient needs were almost always achieved by the performance of the care providers or the facilities and with an optimal quality of care.

None of the treatment attendees complained about inadequate performance in infrastructure or accommodation. The patients' needs were nearly met by the performance of the health facilities with respect to the cost/financial dimension of service (Table 4). With regard to the accessibility dimension of TB service in public health centers, the facilities' service hours and convenience and availability of drugs when required had quality impact values above nine; the corresponding performance scoreswere 0.01 and 0.03 , respectively. These scores means that $1 \%$ to $3 \%$ of treated attendees claimed they received inadequate performance in these aspects of care. Regarding the health information and education dimension of TB care, providing information about the possible side effects of drugs and the importance of the observed treatment had quality impact scores above 9. These values are based on their weighted importance scores of 9.07 and 9.57 and corresponding performances scores of 0.1 and 0.08 , which indicate that only up to $10 \%$ of the respondents, did not receive that particular service in all aspects of care. This result suggests that their needs were nearly met by the performance of the health care providers or health facility. Because the performance scores were between 0.00 and 0.10 and the impact quality scores above 9.0, these aspects of care were of optimal quality from the patient's perspective in the health centers.

Table 4. Optimal quality of TB treatment service from the patient's perspective in hospital, South Ethiopia, March 2012.

\begin{tabular}{|c|c|c|c|c|}
\hline \multirow{2}{*}{ Aspect category } & \multirow{2}{*}{ Quality of TB care aspect } & \multicolumn{3}{|l|}{ Quality score } \\
\hline & & Performance & Importance & Impact \\
\hline \multirow{9}{*}{ Availability and accessibility } & Acceptable waiting times & 0.00 & 6.54 & 10.0 \\
\hline & Service hours of facility convenient & 0.00 & 9.69 & 10.0 \\
\hline & Drugs available when required & 0.00 & 7.08 & 10.0 \\
\hline & Difficulty because of language barrier & 0.00 & 9.54 & 10.0 \\
\hline & Without referral to another health unit for TB services & 0.00 & .00 & 10.0 \\
\hline & TB services available during the working hours & 0.00 & 8.96 & 10.0 \\
\hline & Facility offer services to examine sputum & 0.00 & 10.0 & 10.0 \\
\hline & A treatment observer checking on daily intake of TB drugs & 0.00 & 10.0 & 10.0 \\
\hline & About the possible side effects of TB drugs & 0.04 & 6.46 & 9.64 \\
\hline \multirow{3}{*}{ Information /health education } & The importance of observed treatment & 0.00 & 6.46 & 9.64 \\
\hline & Duration of treatment & 0.04 & 7.23 & 9.64 \\
\hline & TB can be cured & 0.04 & 6.86 & 10.0 \\
\hline \multirow{6}{*}{ Patient provider Interaction } & Care provider treat you with respect & 0.00 & 9.29 & 10.0 \\
\hline & Care providers explain things in an understandable way & 0.00 & 9.86 & 10.0 \\
\hline & Care provider listens carefully & 0.00 & 9.14 & 10.0 \\
\hline & TB-related discrimination & 0.00 & 6.86 & 10.0 \\
\hline & Privacy respected during examination & 0.00 & 10.0 & 10.0 \\
\hline & Care provider showing care and compassion & 0.08 & 10.0 & 9.23 \\
\hline \multirow{4}{*}{$\begin{array}{l}\text { Accommodation of care/ } \\
\text { Infrastructure }\end{array}$} & Facility cleanness & 0.08 & 9.43 & 9.23 \\
\hline & Availability of usable toilets & 0.00 & 9.43 & 10.0 \\
\hline & Availability of Safe drinking water & 0.00 & 7.29 & 10.0 \\
\hline & Enough comfortable benches in weighting area & 0.00 & 9.0 & 10.0 \\
\hline \multirow{4}{*}{ TB-HIV } & Inform about the link between TB and HIV & 0.04 & 9.14 & 9.61 \\
\hline & Inform about how to prevent HIV infection & 0.00 & 10.0 & 10.0 \\
\hline & Was advised to take an HIV test & 0.00 & 10.0 & 10.0 \\
\hline & Informed where to get HIV treatment in case of need & 0.00 & 10.0 & 10.0 \\
\hline \multirow{3}{*}{ Cost or financial issues } & Costs for transport prevented travel to TB facility & 0.00 & 6.68 & 10.0 \\
\hline & Pay for regular TB service & 0.00 & 10.0 & 10.0 \\
\hline & Pay a tip in order to obtain TB services & 0.00 & 2.5 & 10.0 \\
\hline
\end{tabular}

The infrastructure or accommodation of facilities, which involved the availability of usable toilets, had an impact value of 9.31, an importance score of 9.42, and a corresponding performance score of 0.08 . These scores indicated that $8 \%$ of the treated attendees complained of inadequate performance in this care aspect.

Concerning TB-HIV service, providing information on how to prevent HIV infection and advice on how to take an HIV test had a quality impact value above 9, with corresponding performance scores of 0.07 and 0.08 , respectively. This result means that only $7 \%$ and $8 \%$ of treated attendees claimed they received inadequate performance in these aspects of care. Regarding the cost/financial dimension of service, namely obtaining a feefree service and paying tips to obtain the service, this service had quality impact values of 9.92 and 9.90, respectively. This was based on a performance score of 0.01 for all aspects, indicating that nearly none of the respondents were dissatisfied (Table 5). 
Table 5. Optimal quality of TB treatment service from the patient's perspective in public health centers South Ethiopia, March 2012.

\begin{tabular}{lllll}
\hline \multirow{2}{*}{ Aspect category } & Quality of TB care aspect & Quality score & Importance & Impact \\
\cline { 3 - 5 } Availability & Service hours of facility convenient & Performance & 9.01 & 9.92 \\
& Drugs available when required & 0.03 & 9.27 & 9.71 \\
& TB services available during the working hours & 0.03 & 9.63 & 9.71 \\
& About the possible side effects of TB drugs & 0.10 & 9.07 & 9.11 \\
Information /health education & About the importance of observed treatment & 0.08 & 9.57 & 9.25 \\
& About the duration of the TB treatment & 0.07 & 8.92 & 9.33 \\
& About TB can be cured & 0.04 & 9.08 & 9.76 \\
Patient provider Interaction & Care provider treats with respect & 0.04 & 9.14 & 9.69 \\
& Care providers explain things in an understandable way & 0.05 & 9.93 & 9.67 \\
Infrastructure & Sufficient time to discuss & 0.12 & 9.13 & 9.02 \\
TB-HIV & Care provider showing care and compassion & 0.11 & 9.42 & 9.31 \\
& Availability of usable toilets & 0.08 & 9.53 & 9.04 \\
Cost or financial issues & Inform about the link between TB and HIV & 0.11 & 9.38 & 9.42 \\
& Inform about how to prevent HIV infection & 0.07 & 9.48 & 9.27 \\
& Was advised to take an HIV test & 0.08 & 9.13 \\
& Informed where to get HIV treatment in case of need & 0.10 & 9.17 & 9.92 \\
\hline
\end{tabular}

\subsection{Assessing TB Service Satisfaction among TB Patients}

A large proportion $(83.8 \%)$ of TB treatment attendees were satisfied with the services they received. Sociodemographic variables, including marital status, residence, and occupational status, did not appear to be significantly associated with TB patients 'treatment service satisfaction.

\subsection{Institutional Aspects as Predictors of TB Treatment Service Satisfaction}

Multiple linear regression analysis was performed with patient satisfaction variables related to institutional aspects. The model explained $30.4 \%$ of the variation in satisfaction among TB patients. Others factors not addressed in this study may explain the remaining variation in satisfaction with TB care.

Patients who did not have an acceptable waiting time on average decreased the satisfaction score by 1.85 units (95\%CIs: $-2.5,-1.2)$. A treatment observer checking daily intake of TB drugs decreased the satisfaction score by 1.26 units on average (95\%CIs: $-2.27,-.259)$. Furthermore, from the patient-provider interaction aspect, patients had sufficient time to discuss increased the satisfaction score by 1.41 units on average (95\%CIs; $0.54,2.28)$. In addition, patients who did not receive a provider who carefully checked everything during the treatment and examination had an average decrease in their satisfaction score of 1.43 units (95\%CIs: $-2.48,-0.38)$ (Table 6).

Table 6. Multiple linear regression analysis of the predictors of TB treatment service satisfaction at public health facilities, South Ethiopia, 2012

\begin{tabular}{|c|c|c|c|c|c|c|c|}
\hline Institutional explanatory variable & & $\mathbf{N}(\%)$ & Unstandardized B & Std. Error & Standardized B & 95\% CI B & P-value \\
\hline (Constant ßo) & & & 16.02 & .459 & & $15.1,16.9$ & .0001 \\
\hline \multirow{2}{*}{ Waiting time acceptable } & Yes* & $248(67.6)$ & & & & & \\
\hline & No & $119(32.4)$ & -1.85 & .329 & -.34 & $-2.5,-1.2$ & .0001 \\
\hline \multirow{2}{*}{$\begin{array}{l}\text { Treatment observer checking daily intake } \\
\text { of TB drugs }\end{array}$} & No* & $48(13.1)$ & & & & & \\
\hline & Yes & $319(86.9)$ & -1.26 & .512 & -.13 & $-2.3,-.26$ & .014 \\
\hline \multirow{4}{*}{$\begin{array}{l}\text { Costs for transport prevent from getting to } \\
\text { TB facility } \\
\text { Perceived that office has everything needed } \\
\text { to provide complete medical care }\end{array}$} & Yes * & $66(17.9)$ & & & & & \\
\hline & No & $302(82.1)$ & 1.49 & .381 & .23 & $.75,2.25$ & .0001 \\
\hline & No $*$ & $29(8.6)$ & & & & & \\
\hline & Yes & $309(91.4)$ & -1.69 & .637 & -.15 & $-2.95,-.44$ & .008 \\
\hline Experience of paying tip to get TB services & $\begin{array}{l}\text { Yes } \\
\text { No* }\end{array}$ & $\begin{array}{l}4(1.1) \\
364(98.9)\end{array}$ & -3.81 & 1.55 & -.13 & $-6.9,-.8$ & .015 \\
\hline \multirow{2}{*}{ Attended to by the same health provider } & No $*$ & $216(58.4)$ & & & & & \\
\hline & Yes & $154(41.6)$ & 1.13 & .310 & .21 & $.5,1.7$ & .0001 \\
\hline \multirow{2}{*}{$\begin{array}{l}\text { Perceived care providers careful to check } \\
\text { everything when treating and examining }\end{array}$} & Yes* & $311(90.4)$ & & & & & \\
\hline & No & $33(9.6)$ & -1.43 & .535 & -.16 & $-2.48,-.38$ & .008 \\
\hline \multirow{2}{*}{ Sufficient time to discuss } & No $*$ & $323(87.3)$ & & & & & \\
\hline & Yes & $47(12.7)$ & 1.41 & .444 & .185 & $.54,2.28$ & .002 \\
\hline \multirow{2}{*}{ Safe drinking water availability } & No * & $231(62.8)$ & & & & & \\
\hline & Yes & $137(37.2)$ & .82 & .307 & .145 & $.02,1.4$ & .008 \\
\hline \multirow{2}{*}{ Privacy respected during examination } & Yes* & $299(81.2)$ & & & & & \\
\hline & No & $69(18.8)$ & -1.28 & .430 & -.172 & $-2.12,-.43$ & .003 \\
\hline
\end{tabular}

*References category 


\section{Discussion}

This study assessed the quality of TB treatment services in public health facilities from the patient's perspective. In this study, a large proportion of TB treatment attendees were satisfied with the TB treatment services; $83.8 \%$ reported that they were totally satisfied with TB treatment services.

A previous comparable study on TB treatment attendees performed in Afar, Ethiopia, showed a relatively lower proportion of patients satisfied with TB services $(70.3 \%)$ [18], as did another study performed by Zewdie et al. [14] in Central Shewa, Ethiopia (62.6\%). This might be due to differences in the study population and setting or that the size of the TB treatment attendance facilities was small compared to other service clients. Small facilities might enable providers to provide more attention and, in turn, improve interactions with treatment attendees. In addition, a higher satisfaction rate in this study could be attributed to the reluctance of patients to express their dissatisfaction with the services they received, since the interview for this study was undertaken within the facilities themselves and they may have felt that a negative response would prejudice treatment.

In this study, socio-demographic variables (marital status, residence, and occupational status) were not significantly associated with overall satisfaction of the TB treatment service. This result is similar to a study performed in Alexandria, Egypt, where the socio-demographic variables age and sex did not significantly predict the satisfaction of TB treatment attendees [19]. In contrast, in a study performed in central Shewa, marital status, residence, educational status, and occupational status appeared to be significantly associated with satisfaction, and likewise in a similar study in India, health status and being married were positively associated with patient satisfaction [14, 20]. These differences might be attributed to variations in scope, study setting, and study period.

Our findings demonstrate that patients who did not have an acceptable waiting time averaged a decrease of 1.85 units in their satisfaction score (95\%CIs: -2.5 to -1.2$)$. This is in line with other studies in Ghana, where the waiting time was found to be inversely and significantly related to consumer satisfaction and is supported by the qualitative finding that short waiting time before service rose as reason for patient satisfaction [21].

Providing treatment using a directly observed method daily during the first two months is a DOT component strategy; a treatment observer checking daily intake of TB drugs averaged a decrease in 1.26 units in satisfaction scores (95\%CIs: -2.27 to -.26). In addition, from the patient provider interaction domain, patients having sufficient time to discuss increased satisfaction scores by an average of 1.41 units (95\%CIs: 0.54 to 2.28). These findings are supported by studies previously performed in central Shewa, which showed that very short duration of stay with the provider reduced the satisfaction score by 0.36 units. In addition, respondents not perceiving the provider carefully checking everything when treating and examining resulted in an average decrease in satisfaction scores of 1.43 units (95\%CIs: -2.48 to -0.38 ). Similarly, patient disagreement with technical competency had greater negative influence on patient satisfaction in the same study [14].

\section{Conclusions}

This study showed that the majority of TB treatment attendees were satisfied with the TB service provided in public health facilities. The perceived performance of TBcare aspects that independently predicted overall satisfaction were waiting time, checking the daily intake of TB drugs by a treatment observer, paying a tip to obtain TB services, being attended to by the same health provider, noticing that care providers were careful to check everything during treatment and examination, and having sufficient time for discussion.

Inadequate aspects of quality of TB-care reported by patients were: a notable gap between their expectations and their experiences in public health centers, most aspects of provided care in the domain of availability, information/communication of TB care, and patientprovider interaction and infrastructure/basic amenities of public health facilities.

An optimal quality of TB care was found in all public health facilities, or there was no gap between patients' expectations and their experiences in fee-free service at the point of service delivery and TB-HIV integration services. These aspects were perceived as having no room for improvement from a patient's perspective.

\section{Conflict of interests}

The authors declare that they have no competing interests.

There is no conflict of interest between the funding office and authors.

\section{Authors' Contributions}

All the authors were involved in the conception, design, study conduct, analysis and interpretation of the findings. The authors have read and approved the final content of the manuscript.

\section{Acknowledgements}

We are grateful to Jima University for funding this research. The authors gratefully acknowledge editorial assistance from the Nextgenediting Global Initiative. 


\section{References}

[1] World Health Organization: Implementing the Stop TB Strategy a Handbook for national Tuberculosis control programmes. Geneva: World Health Organization; 2008.

[2] Katherine MB, Nathaniel S, Rhett S, Loice A, and Philip K: A quality assessment tool for tuberculosis control activities in resource limited settings. Tuberculosis (Edinb) 2011:12.

[3] World Health Organization: Global tuberculosis control Report 2011. In.; 2011.

[4] JAN MAINZ: Defining and classifying clinical indicators for quality improvement. International Journal for Quality in Health Care 2003, 15(6):523-530.

[5] Federal Ministry of Health: Health Sector Strategic Plan (HSDP-III). In. Edited by Programming HPa, vol. 3. Addis Ababa: FMOH; 2009.

[6] Maher D, Blanc L, Raviglione M: WHO policies for tuberculosis control. Lancet 2004:363-1911.

[7] Federal Ministry of Health: Tuberculosis, Leprosy and TB/HIV Prevention and Control Programme Manual. In. Edited by Control THPa, 4 edn. Addis Ababa; 2008.

[8] Mengiste MM, James NN, John DW, Amanuel G, Tassew T, Frew L, and et'al: Quality of tuberculosis care and its association with patient adherence to treatment in eight Ethiopian districts. Health Policy and Planning 2009, 24:457-466.

[9] Borgdorff MW, Floyd K, Broekmans JF: Interventions to reduce tuberculosis mortality and transmission in low- and middle-income countries. Bull. World Health Organ. [PubMed: 11984608] 2002, 80(3):217-227.

[10] Shargie EB, Lindtjorn B: Determinants of treatment adherence among smear-positive tuberculosis patients in Southern Ethiopia. PLOS medicine PubMed 2007, 4:280287.
[11] Jin BW, Kim SC, Mori T, Shimao T: The impact of intensified supervisory activities on tuberculosis treatment, Tubercle and Lung Disease 1993, 74:267-272.

[12] Ovretveit J: Health service quality: An introduction to quality methods for health services. London: Blackwell Scientific; 1992.

[13] Hadiya Zonl Health Department: Hadiya Zone Health Department anuual report In. Hossana; 2011

[14] Birhanu Z, Assefa T, Woldie M, Morankar S: Determinants of satisfaction with health care provider interactions at health centres in central Ethiopia. BMC Health Services Research 2010, 13(74):1472-6963.

[15] Grant N, Marshall, Ron DH: The patient satisfaction Questionnaire short- form (PSQ-18), RAND, Santa Monica, CA. 1994.

[16] Tuberculosis Coalition for Technical Assistance, QUOTE TB. Guide for improving TB care as seen through the eyes of the patient. Amsterdam: Tuberculosis Coalition for Technical Assistance; 2007.

[17] Herman J, Sixma, Jan JK, Crétien vC, Peters L: Quality of care from the patients' perspective: from theoretical concept to a new measuring instrument: Health Expectations; 1998.

[18] Girma A, H/Mariam D, Deribe K: Quality of tuberculosis care in six health facilities of Afar Region, Ethiopia. Ethiop Med J 2010, 48(3):195-202.

[19] Kamel MI, Rashed S, Foda N, Mohie A, Loutfy M: Gender differences in health care utilization and outcome of respiratory tuberculosis in Alexandria Eastern Mediterranean Health Journal 2003, 9(4).

[20] Aditi N: Factors affecting patient satisfaction and healthcare quality. International Journal of Health Care Quality Assurance 2009, 22(4):366-381.

[21] Edward NA: Determinants of Consumer Satisfaction of Health Care in Ghana: Does Choice of Health Care Provider Matter? Global journal of health science 2009, 1(2). 